\title{
INVARIANCE PROPERTIES OF AN OPERATOR PRODUCT INVOLVING GENERALIZED INVERSES*
}

\author{
ZHIPING XIONG ${ }^{\dagger}$ AND YINGYING QIN Q $^{\ddagger}$
}

\begin{abstract}
Given bounded linear operators $T_{1}, T_{2}$ and $T_{3}$, this paper investigates certain invariance properties of the operator product $T_{1} X T_{3}$ with respect to the choice of bounded linear operator $X$, where $X$ is a generalized inverse of $T_{2}$. Different types of generalized inverses are taken into account.
\end{abstract}

Key words. Bounded linear operators, Generalized inverse, Moore-Penrose inverse, Operator product, Invariance property.

AMS subject classifications. 15A09, 15A24, 47A05.

1. Introduction. Throughout this paper, $\mathbb{H}, \mathbb{K}$, and $\mathbb{L}$ denote arbitrary Hilbert spaces. We use $L(\mathbb{H}, \mathbb{K})$ to denote the set of all bounded linear operators from $\mathbb{H}$ to $\mathbb{K}$. Also, $L(\mathbb{H})=L(\mathbb{H}, \mathbb{H})$. I denotes the identity operator on Hilbert spaces and $O$ is the zero operator on Hilbert spaces. For $T \in L(\mathbb{H}, \mathbb{K})$, the symbols $T^{*}, R(T)$, and $N(T)$ will stand for the adjoint operator, the range, and the kernel of $T$, respectively.

Let $T \in L(\mathbb{H}, \mathbb{K})$. If there exists an operator $X \in L(\mathbb{K}, \mathbb{H})$ satisfying the following four operator equations:
(1) $T X T=T$,
(2) $X T X=X$,
(3) $(T X)^{*}=T X$,
(4) $(X T)^{*}=X T$,

then $X$ is called a Moore-Penrose inverse of $T$ and denoted by $T^{\dagger}$. As we know, $T$ has a Moore-Penrose inverse if and only if $R(T)$ is closed and the Moore-Penrose inverse of $T$ is unique (see, for example, $[5,7,14,17,18,19,20,21,22,23]$ ). For a subset $\eta \subseteq\{1,2,3,4\}$, the set of operators satisfying the equations contained in $\eta$ is denoted by $T \eta$. An operator from $T \eta$ is called an $\eta$-inverse of $T$. For example, an operator $X$ of the set $T\{1\}$ is called a $\{1\}$-inverse of $T$ and denoted by $T^{(1)}$ or $T^{-}$. One usually denotes any $\{1,3\}$-inverse of $T$ as $T^{(1,3)}$ and any $\{1,4\}$-inverse of $T$ is denoted by $T^{(1,4)}$. The unique $\{1,2,3,4\}$-inverse of $T$ is the Moore-Penrose inverse of $T$. We

*Received by the editors on April 16, 2011. Accepted for publication on June 27, 2011. Handling Editor: Oskar Maria Baksalary.

${ }^{\dagger}$ Department of Mathematics, Wuyi University, Jiangmen 529020, Guangdong, P.R. China (xzpwhere@163.com). The work was supported by the start-up fund of Wuyi University, Jiangmen 529020, Guangdong Province.

$\ddagger$ Department of Mathematics, Wuyi University, Jiangmen 529020, Guangdong, P.R. China (qinyy04@163.com). 
refer the reader to $[5,6,9,10,11,15,16,23,24]$ for basic results on the generalized inverses of operators.

Invariance properties of operator product involving generalized inverses are fundamental in the theory of operators. They have attracted considerable attention and many interesting results have been obtained (see, for example, $[1,2,3,4,12,13]$ ). In this paper, given bounded linear operators $T_{1} \in L(\mathbb{L}, \mathbb{H}), T_{2} \in L(\mathbb{L}, \mathbb{K})$ and $T_{3} \in L(\mathbb{H}, \mathbb{K})$, we investigate properties of the operator product $T_{1} T_{2}^{-} T_{3}$ for various types of generalized inverses $T_{2}^{-}$of $T_{2}$, where our interest is focused on invariance properties with respect to the choice of $T_{2}^{-}$concerning the value and range of $T_{1} T_{2}^{-} T_{3}$.

We first mention the following three results, which will be used in this paper.

Lemma 1.1. [9]. Let $T \in L(\mathbb{H}, \mathbb{K})$ have a closed range. Then

$$
T\{1\}=\left\{T^{\dagger}+Y-T^{\dagger} T Y T T^{\dagger}: \quad Y \in L(\mathbb{K}, \mathbb{H})\right\} .
$$

Lemma 1.2. $[8,9]$. Let $T \in L(\mathbb{H}, \mathbb{K})$ have a closed range and $X \in L(\mathbb{K}, \mathbb{H})$. Then the following statements are equivalent:

(1) $T X T=T$ and $(T X)^{*}=T X$;

(2) there exists some $Y \in L(\mathbb{K}, \mathbb{H})$ such that $X=T^{\dagger}+\left(I-T^{\dagger} T\right) Y$.

Lemma 1.3. $[8,9]$. Let $T \in L(\mathbb{H}, \mathbb{K})$ have a closed range and $X \in L(\mathbb{K}, \mathbb{H})$. Then the following statements are equivalent:

(1) $T X T=T$ and $(X T)^{*}=X T$;

(2) there exists some $Y \in L(\mathbb{K}, \mathbb{H})$ such that $X=T^{\dagger}+Y\left(I-T T^{\dagger}\right)$.

2. Invariance properties of operator product $T_{1} T_{2}^{(1)} T_{3}$. Let $T_{1} \in L(\mathbb{L}, \mathbb{H})$, $T_{2} \in L(\mathbb{L}, \mathbb{K})$ and $T_{3} \in L(\mathbb{H}, \mathbb{K})$ be such that $T_{1}, T_{2}$ and $T_{3}$ have closed ranges. In this section, we will study several invariance properties of the operator product $T_{1} T_{2}^{(1)} T_{3}$ with respect to the choice of $T_{2}^{(1)} \in T_{2}\{1\}$. The main result is the following theorem.

TheOREm 2.1. Let $T_{1} \in L(\mathbb{L}, \mathbb{H}), T_{2} \in L(\mathbb{L}, \mathbb{K})$ and $T_{3} \in L(\mathbb{H}, \mathbb{K})$ be such that $T_{1}, T_{2}, T_{3}$ have closed ranges. Suppose that $T_{1}, T_{2}$, and $T_{3}$ are not zero operators on Hilbert spaces. Then the following statements are equivalent:

(1) The operator product $T_{1} T_{2}^{(1)} T_{3}$ does not depend on the choice of $T_{2}^{(1)} \in$ $T_{2}\{1\}$ 
(2) $R\left(T_{1}^{*}\right) \subseteq R\left(T_{2}^{*}\right)$ and $R\left(T_{3}\right) \subseteq R\left(T_{2}\right)$.

Proof. According to the technique of block operator matrices in [7], we know that the operator $T_{2}$ has the following matrix form with respect to the orthogonal sum of subspaces:

$$
T_{2}=\left(\begin{array}{cc}
T_{2}^{11} & O \\
O & O
\end{array}\right):\left(\begin{array}{c}
R\left(T_{2}^{*}\right) \\
N\left(T_{2}\right)
\end{array}\right) \rightarrow\left(\begin{array}{c}
R\left(T_{2}\right) \\
N\left(T_{2}^{*}\right)
\end{array}\right)
$$

where $T_{2}^{11}$ is invertible in $L\left(R\left(T_{2}^{*}\right), R\left(T_{2}\right)\right)$, and

$$
T_{2}^{\dagger}=\left(\begin{array}{cc}
\left(T_{2}^{11}\right)^{-1} & O \\
O & O
\end{array}\right):\left(\begin{array}{c}
R\left(T_{2}\right) \\
N\left(T_{2}^{*}\right)
\end{array}\right) \rightarrow\left(\begin{array}{c}
R\left(T_{2}^{*}\right) \\
N\left(T_{2}\right)
\end{array}\right)
$$

Also we have that the operator $T_{1}$ has the following form:

$$
T_{1}=\left(\begin{array}{cc}
T_{1}^{11} & T_{1}^{12} \\
O & O
\end{array}\right):\left(\begin{array}{c}
R\left(T_{2}^{*}\right) \\
N\left(T_{2}\right)
\end{array}\right) \rightarrow\left(\begin{array}{c}
R\left(T_{1}\right) \\
N\left(T_{1}^{*}\right)
\end{array}\right)
$$

and

$$
T_{1}^{*}=\left(\begin{array}{cc}
\left(T_{1}^{11}\right)^{*} & O \\
\left(T_{1}^{12}\right)^{*} & O
\end{array}\right):\left(\begin{array}{c}
R\left(T_{1}\right) \\
N\left(T_{1}^{*}\right)
\end{array}\right) \rightarrow\left(\begin{array}{c}
R\left(T_{2}^{*}\right) \\
N\left(T_{2}\right)
\end{array}\right)
$$

and

$$
T_{1} T_{1}^{*}=\left(\begin{array}{cc}
D & O \\
O & O
\end{array}\right):\left(\begin{array}{c}
R\left(T_{1}\right) \\
N\left(T_{1}^{*}\right)
\end{array}\right) \rightarrow\left(\begin{array}{c}
R\left(T_{1}\right) \\
N\left(T_{1}^{*}\right)
\end{array}\right)
$$

where $D=T_{1}^{11}\left(T_{1}^{11}\right)^{*}+T_{1}^{12}\left(T_{1}^{12}\right)^{*}$ is positive and invertible in $L\left(R\left(T_{1}\right)\right)$. In particular

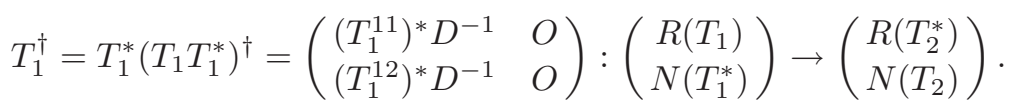

Furthermore, we obtain that the operator $T_{3}$ has the following matrix form with respect to the orthogonal sum of subspaces:

$$
T_{3}=\left(\begin{array}{cc}
T_{3}^{11} & O \\
T_{3}^{21} & O
\end{array}\right):\left(\begin{array}{c}
R\left(T_{3}^{*}\right) \\
N\left(T_{3}\right)
\end{array}\right) \rightarrow\left(\begin{array}{c}
R\left(T_{2}\right) \\
N\left(T_{2}^{*}\right)
\end{array}\right)
$$

and

$$
T_{3}^{*}=\left(\begin{array}{cc}
\left(T_{3}^{11}\right)^{*} & \left(T_{3}^{21}\right)^{*} \\
O & O
\end{array}\right):\left(\begin{array}{c}
R\left(T_{2}\right) \\
N\left(T_{2}^{*}\right)
\end{array}\right) \rightarrow\left(\begin{array}{c}
R\left(T_{3}^{*}\right) \\
N\left(T_{3}\right)
\end{array}\right)
$$

and

$$
T_{3}^{*} T_{3}=\left(\begin{array}{cc}
S & O \\
O & O
\end{array}\right):\left(\begin{array}{c}
R\left(T_{3}^{*}\right) \\
N\left(T_{3}\right)
\end{array}\right) \rightarrow\left(\begin{array}{c}
R\left(T_{3}^{*}\right) \\
N\left(T_{3}\right)
\end{array}\right),
$$


where $S=\left(T_{3}^{11}\right)^{*} T_{3}^{11}+\left(T_{3}^{21}\right)^{*} T_{3}^{21}$ is positive and invertible in $L\left(R\left(T_{3}^{*}\right)\right)$. Then

(2.6) $T_{3}^{\dagger}=\left(T_{3}^{*} T_{3}\right)^{\dagger} T_{3}^{*}=\left(\begin{array}{cc}S^{-1}\left(T_{3}^{11}\right)^{*} & S^{-1}\left(T_{3}^{21}\right)^{*} \\ O & O\end{array}\right):\left(\begin{array}{c}R\left(T_{2}\right) \\ N\left(T_{2}^{*}\right)\end{array}\right) \rightarrow\left(\begin{array}{c}R\left(T_{3}^{*}\right) \\ N\left(T_{3}\right)\end{array}\right)$.

Next, we will prove the facts that (1) and (2) in Theorem 2.1 are equivalent.

$(2) \Rightarrow(1)$ : The inclusion $R\left(T_{1}^{*}\right) \subseteq R\left(T_{2}^{*}\right)$ is equivalent to $T_{1} T_{2}^{\dagger} T_{2}=T_{1}$. Then from (2.1)-(2.6), we have

$$
T_{1} T_{2}^{\dagger} T_{2}=\left(\begin{array}{cc}
T_{1}^{11} & T_{1}^{12} \\
O & O
\end{array}\right)\left(\begin{array}{cc}
\left(T_{2}^{11}\right)^{-1} & O \\
O & O
\end{array}\right)\left(\begin{array}{cc}
T_{2}^{11} & O \\
O & O
\end{array}\right)=\left(\begin{array}{cc}
T_{1}^{11} & O \\
O & O
\end{array}\right) .
$$

Hence, according to (2.3) and (2.7), the equality $T_{1} T_{2}^{\dagger} T_{2}=T_{1}$ is equivalent to $T_{1}^{12}=$ $O$, that is

$$
R\left(T_{1}^{*}\right) \subseteq R\left(T_{2}^{*}\right) \Leftrightarrow T_{1}^{12}=O
$$

In the same manner, we can prove that the inclusion $R\left(T_{3}\right) \subseteq R\left(T_{2}\right)$ is equivalent to $T_{2} T_{2}^{\dagger} T_{3}=T_{3}$ and

$$
R\left(T_{3}\right) \subseteq R\left(T_{2}\right) \Leftrightarrow T_{3}^{21}=O
$$

On the other hand, from Lemma 1.1 it follows that arbitrary $T_{2}^{(1)} \in T_{2}\{1\}$ has the form

$$
T_{2}^{(1)}=\left(\begin{array}{cc}
\left(T_{2}^{11}\right)^{-1} & U \\
V & W
\end{array}\right)
$$

where $U, V$ and $W$ are bounded linear operators on appropriate spaces. Hence, from (2.2), (2.3), (2.5), (2.8), (2.9), (2.10), we have that

$$
T_{1} T_{2}^{(1)} T_{3}=\left(\begin{array}{cc}
T_{1}^{11}\left(T_{2}^{11}\right)^{-1} T_{3}^{11} & O \\
O & O
\end{array}\right)=T_{1} T_{2}^{\dagger} T_{3}
$$

Combining (2.10) with (2.11), we have the result $(2) \Rightarrow(1)$.

$(1) \Rightarrow(2)$ : Since the Moore-Penrose inverse of a bounded linear operator is unique and belongs to the set of $\{1\}$-inverse, it is clear that $T_{1} T_{2}^{(1)} T_{3}$ doesn't depend on the choice of $T_{2}^{(1)} \in T_{2}\{1\}$ if and only if the equality $T_{1} T_{2}^{(1)} T_{3}=T_{1} T_{2}^{\dagger} T_{3}$ holds for every $T_{2}^{(1)} \in T_{2}\{1\}$. By Lemma 1.1, it follows that both

$$
M=\left(\begin{array}{cc}
\left(T_{2}^{11}\right)^{-1} & \left(T_{1}^{11}\right)^{*} \\
O & \left(T_{1}^{12}\right)^{*}
\end{array}\right) \text { and } N=\left(\begin{array}{cc}
\left(T_{2}^{11}\right)^{-1} & O \\
\left(T_{3}^{11}\right)^{*} & \left(T_{3}^{21}\right)^{*}
\end{array}\right)
$$

are $\{1\}$-inverses of $T_{2}$. Since

$$
T_{1} M T_{3}=T_{1} N T_{3}=T_{1} T_{2}^{\dagger} T_{3}=\left(\begin{array}{cc}
T_{1}^{11}\left(T_{2}^{11}\right)^{-1} T_{3}^{11} & O \\
O & O
\end{array}\right)
$$


from $(2.2),(2.3),(2.5),(2.12),(2.13)$, we get

$$
T_{1}^{11}\left(T_{1}^{11}\right)^{*} T_{3}^{21}+T_{1}^{12}\left(T_{1}^{12}\right)^{*} T_{3}^{21}=O
$$

and

$$
T_{1}^{12}\left(T_{3}^{11}\right)^{*} T_{3}^{11}+T_{1}^{12}\left(T_{3}^{21}\right)^{*}\left(T_{3}^{21}\right)=O .
$$

Combining (2.14), (2.15) with the equalities (2.3) and (2.5), we have

$$
D T_{3}^{21}=O \text { and } T_{1}^{12} S=O .
$$

Since $D$ and $S$ are invertible, from (2.16) we obtain

$$
T_{3}^{21}=O \text { and } T_{1}^{12}=O,
$$

which are respectively equivalent to $R\left(T_{3}\right) \subseteq R\left(T_{2}\right)$ and $R\left(T_{1}^{*}\right) \subseteq R\left(T_{2}^{*}\right)$.

Corollary 2.2. Let $T_{1} \in L(\mathbb{L}, \mathbb{H}), T_{2} \in L(\mathbb{L}, \mathbb{K})$, and $T_{3} \in L(\mathbb{H}, \mathbb{K})$ be such that $T_{1}, T_{2}, T_{3}$ have closed ranges. Suppose that $T_{1}, T_{2}$, and $T_{3}$ are not zero operators on Hilbert spaces. Then the identity $T_{1} T_{2}^{(1)} T_{3}=O$ holds for every $T_{2}^{(1)} \in T_{2}\{1\}$ if and only if $R\left(T_{1}^{*}\right) \subseteq R\left(T_{2}^{*}\right), R\left(T_{3}\right) \subseteq R\left(T_{2}\right)$, and $R\left(T_{3}\right) \subseteq N\left(T_{1} T_{2}^{\dagger}\right)$.

Corollary 2.3. Let $T_{1} \in L(\mathbb{L}, \mathbb{H}), T_{2} \in L(\mathbb{L}, \mathbb{K})$, and $T_{3} \in L(\mathbb{H}, \mathbb{K})$ be such that $T_{1}, T_{2}, T_{3}$ have closed ranges. If $R\left(T_{1}^{*}\right) \subseteq R\left(T_{2}^{*}\right)$ and $R\left(T_{3}\right) \subseteq R\left(T_{2}\right)$, then $R\left(T_{1} T_{2}^{(1)} T_{3}\right)$ is the same for every $T_{2}^{(1)} \in T_{2}\{1\}$.

3. Invariance properties of products $T_{1} T_{2}^{(1,3)} T_{3}$ and $T_{1} T_{2}^{(1,4)} T_{3}$. Let $T_{1} \in$ $L(\mathbb{L}, \mathbb{H}), T_{2} \in L(\mathbb{L}, \mathbb{K})$, and $T_{3} \in L(\mathbb{H}, \mathbb{K})$. In this section, we will investigate the invariance properties of the operator products $T_{1} T_{2}^{(1,3)} T_{3}$ and $T_{1} T_{2}^{(1,4)} T_{3}$ with respect to any $T_{2}^{(1,3)} \in T_{2}\{1,3\}$ and $T_{2}^{(1,4)} \in T_{2}\{1,4\}$.

Theorem 3.1. Let $T_{1} \in L(\mathbb{L}, \mathbb{H}), T_{2} \in L(\mathbb{L}, \mathbb{K})$, and $T_{3} \in L(\mathbb{H}, \mathbb{K})$ be such that $T_{1}, T_{2}, T_{3}$ have closed ranges. Suppose that $T_{1}, T_{2}$, and $T_{3}$ are not zero operators on Hilbert spaces. Then the following statements are equivalent:

(1) The equality $T_{1} T_{2}^{(1,3)} T_{3}=T_{1} T_{2}^{\dagger} T_{3}$ holds for every $T_{2}^{(1,3)} \in T_{2}\{1,3\}$;

(2) $R\left(T_{1}^{*}\right) \subseteq R\left(T_{2}^{*}\right)$.

Proof. By Lemma 1.2, we have that arbitrary $T_{2}^{(1,3)} \in T_{2}\{1,3\}$ has the form

$$
T_{2}^{(1,3)}=\left(\begin{array}{cc}
\left(T_{2}^{11}\right)^{-1} & O \\
W_{21} & W_{22}
\end{array}\right)
$$

where $W_{21}$ and $W_{22}$ are bounded linear operators on appropriate subspaces. Then from the equalities $(2.2),(2.3),(2.5)$ and $(3.1)$, we know that for any $T_{2}^{(1,3)} \in T_{2}\{1,3\}$

$$
T_{1} T_{2}^{(1,3)} T_{3}=\left(\begin{array}{cc}
T_{1}^{11} & T_{1}^{12} \\
O & O
\end{array}\right)\left(\begin{array}{cc}
\left(T_{2}^{11}\right)^{-1} & O \\
W_{21} & W_{22}
\end{array}\right)\left(\begin{array}{cc}
T_{3}^{11} & O \\
T_{3}^{21} & O
\end{array}\right)
$$




$$
=\left(\begin{array}{cc}
T_{1}^{11}\left(T_{2}^{11}\right)^{-1} T_{3}^{11}+T_{1}^{12} W_{21} T_{3}^{11}+T_{1}^{12} W_{22} T_{3}^{21} & O \\
O & O
\end{array}\right)
$$

and

$$
T_{1} T_{2}^{\dagger} T_{3}=\left(\begin{array}{cc}
T_{1}^{11}\left(T_{2}^{11}\right)^{-1} T_{3}^{11} & O \\
O & O
\end{array}\right)
$$

We now prove that (1) and (2) in Theorem 3.1 are equivalent.

$(2) \Rightarrow(1)$ : Since

$$
R\left(T_{1}^{*}\right) \subseteq R\left(T_{2}^{*}\right) \Leftrightarrow T_{1}^{12}=O
$$

we have that for arbitrary $T_{2}^{(1,3)} \in T_{2}\{1,3\}$,

$$
\begin{aligned}
T_{1} T_{2}^{(1,3)} T_{3} & =\left(\begin{array}{cc}
T_{1}^{11}\left(T_{2}^{11}\right)^{-1} T_{3}^{11}+T_{1}^{12} W_{21} T_{3}^{11}+T_{1}^{12} W_{22} T_{3}^{21} & O \\
O & O
\end{array}\right) \\
& =\left(\begin{array}{cc}
T_{1}^{11}\left(T_{2}^{11}\right)^{-1} T_{3}^{11} & O \\
O & O
\end{array}\right) \\
& =T_{1} T_{2}^{\dagger} T_{3} .
\end{aligned}
$$

$(1) \Rightarrow(2)$ : From Lemma 1.2, it follows that

$$
M_{1}=\left(\begin{array}{cc}
\left(T_{2}^{11}\right)^{-1} & O \\
\left(T_{3}^{11}\right)^{*} & \left(T_{3}^{21}\right)^{*}
\end{array}\right)
$$

is a $\{1,3\}$-inverses of $T_{2}$. Then

$$
\begin{aligned}
T_{1} M_{1} T_{3} & =\left(\begin{array}{cc}
T_{1}^{11} & T_{1}^{12} \\
O & O
\end{array}\right)\left(\begin{array}{cc}
\left(T_{2}^{11}\right)^{-1} & O \\
\left(T_{3}^{11}\right)^{*} & \left(T_{3}^{21}\right)^{*}
\end{array}\right)\left(\begin{array}{cc}
T_{3}^{11} & O \\
T_{3}^{21} & O
\end{array}\right) \\
& =\left(\begin{array}{ccc}
T_{1}^{11}\left(T_{2}^{11}\right)^{-1} T_{3}^{11}+T_{1}^{12}\left(T_{3}^{11}\right)^{*} T_{3}^{11}+T_{1}^{12}\left(T_{3}^{21}\right)^{*} T_{3}^{21} & O \\
O & O
\end{array}\right)
\end{aligned}
$$

and

$$
T_{1} M_{1} T_{3}=T_{1} T_{2}^{\dagger} T_{3}=\left(\begin{array}{cc}
T_{1}^{11}\left(T_{2}^{11}\right)^{-1} T_{3}^{11} & O \\
O & O
\end{array}\right) .
$$

Hence, from (3.3) and (3.4), we have

$$
T_{1}^{12}\left(T_{3}^{11}\right)^{*} T_{3}^{11}+T_{1}^{12}\left(T_{3}^{21}\right)^{*} T_{3}^{21}=O
$$

Combining (3.5) with the equality (2.5), we have $T_{1}^{12} S=O$. Since $S$ is invertible, $T_{1}^{12}=O$, that is $R\left(T_{1}^{*}\right) \subseteq R\left(T_{2}^{*}\right) . \square$ 
Corollary 3.2. Let $T_{1} \in L(\mathbb{L}, \mathbb{H}), T_{2} \in L(\mathbb{L}, \mathbb{K})$, and $T_{3} \in L(\mathbb{H}, \mathbb{K})$ be such that $T_{1}, T_{2}, T_{3}$ have closed ranges. Suppose that $T_{1}, T_{2}$, and $T_{3}$ are not zero operators on Hilbert spaces. Then the identity $T_{1} T_{2}^{(1,3)} T_{3}=O$ holds for every $T_{2}^{(1,3)} \in T_{2}\{1,3\}$ if and only if $R\left(T_{1}^{*}\right) \subseteq R\left(T_{2}^{*}\right)$ and $R\left(T_{3}\right) \subseteq N\left(T_{1} T_{2}^{\dagger}\right)$.

Next, we will investigate the invariance of the range of the operator product $T_{1} T_{2}^{(1,3)} T_{3}$ with respect to the choices of $T_{2}^{(1,3)} \in T_{2}\{1,3\}$.

Theorem 3.3. Let $T_{1} \in L(\mathbb{L}, \mathbb{H}), T_{2} \in L(\mathbb{L}, \mathbb{K})$, and $T_{3} \in L(\mathbb{H}, \mathbb{K})$ be such that $T_{1}, T_{2}, T_{3}$ have closed ranges. Suppose that $T_{1}, T_{2}$, and $T_{3}$ are not zero operators on Hilbert spaces and $T_{1} T_{2}^{\dagger} T_{3} \neq O$. Then the following statements are equivalent:

(1) $R\left(T_{1} T_{2}^{(1,3)} T_{3}\right)$ is the same for every $T_{2}^{(1,3)} \in T_{2}\{1,3\}$;

(2) $R\left(T_{1}^{*}\right) \subseteq R\left(T_{2}^{*}\right)$.

Proof. According to the above proof in Theorem 3.1, it follows that for any $T_{2}^{(1,3)} \in T_{2}\{1,3\}$

$$
T_{1} T_{2}^{(1,3)} T_{3}=\left(\begin{array}{cc}
T_{1}^{11}\left(T_{2}^{11}\right)^{-1} T_{3}^{11}+T_{1}^{12} W_{21} T_{3}^{11}+T_{1}^{12} W_{22} T_{3}^{21} & O \\
O & O
\end{array}\right)
$$

and

$$
\left(T_{1} T_{2}^{(1,3)} T_{3}\right)^{\dagger}=\left(\begin{array}{cc}
\left(T_{1}^{11}\left(T_{2}^{11}\right)^{-1} T_{3}^{11}+T_{1}^{12} W_{21} T_{3}^{11}+T_{1}^{12} W_{22} T_{3}^{21}\right)^{\dagger} & O \\
O & O
\end{array}\right)
$$

where $W_{21}$ and $W_{22}$ are bounded linear operators on appropriate subspaces. Furthermore, from (3.2) we have

$$
\left(T_{1} T_{2}^{\dagger} T_{3}\right)^{\dagger}=\left(\begin{array}{cc}
\left(T_{1}^{11}\left(T_{2}^{11}\right)^{-1} T_{3}^{11}\right)^{\dagger} & O \\
O & O
\end{array}\right)
$$

$(2) \Rightarrow(1)$ : Clearly the invariance of the product $T_{1} T_{2}^{(1,3)} T_{3}$ with respect to $T_{2}^{(1,3)} \in$ $T_{2}\{1,3\}$ is sufficient for the invariance of $R\left(T_{1} T_{2}^{(1,3)} T_{3}\right)$ with respect to the choices of $T_{2}^{(1,3)} \in T_{2}\{1,3\}$. Then from Theorem 3.1, we have the result " $(2) \Rightarrow(1)$ " in Theorem 3.3 .

$(1) \Rightarrow(2)$ : From (1) in Theorem 3.3, we know that the equality $R\left(T_{1} T_{2}^{(1,3)} T_{3}\right)=$ $R\left(T_{1} T_{2}^{\dagger} T_{3}\right)$ holds for any $T_{2}^{(1,3)} \in T_{2}\{1,3\}$. Under the definition of the range of operators, it follows that the equality $R\left(T_{1} T_{2}^{(1,3)} T_{3}\right)=R\left(T_{1} T_{2}^{\dagger} T_{3}\right)$ holds for any $T_{2}^{(1,3)}$ if and only if the following two inclusions:

$$
R\left(T_{1} T_{2}^{(1,3)} T_{3}\right) \subseteq R\left(T_{1} T_{2}^{\dagger} T_{3}\right)
$$

and

$$
R\left(T_{1} T_{2}^{\dagger} T_{3}\right) \subseteq R\left(T_{1} T_{2}^{(1,3)} T_{3}\right)
$$


hold for any $T_{2}^{(1,3)} \in T_{2}\{1,3\}$, which are respectively equivalent to the following two identities:

$$
T_{1} T_{2}^{\dagger} T_{3}\left(T_{1} T_{2}^{\dagger} T_{3}\right)^{\dagger} T_{1} T_{2}^{(1,3)} T_{3}=T_{1} T_{2}^{(1,3)} T_{3}
$$

and

$$
T_{1} T_{2}^{(1,3)} T_{3}\left(T_{1} T_{2}^{(1,3)} T_{3}\right)^{\dagger} T_{1} T_{2}^{\dagger} T_{3}=T_{1} T_{2}^{\dagger} T_{3}
$$

valid for any $T_{2}^{(1,3)} \in T_{2}\{1,3\}$. Hence, from the equalities (3.2), (3.6), (3.7), (3.8) (3.9), (3.10), we have

$$
\left(T_{1}^{11}\left(T_{2}^{11}\right)^{-1} T_{3}^{11}\right)\left(T_{1}^{11}\left(T_{2}^{11}\right)^{-1} T_{3}^{11}\right)^{\dagger} \mu=\mu
$$

and

$$
\mu \mu^{\dagger} T_{1}^{11}\left(T_{2}^{11}\right)^{-1} T_{3}^{11}=T_{1}^{11}\left(T_{2}^{11}\right)^{-1} T_{3}^{11}
$$

where $W_{21}$ and $W_{22}$ are bounded linear operators on appropriate subspaces and

$$
\mu=T_{1}^{11}\left(T_{2}^{11}\right)^{-1} T_{3}^{11}+T_{1}^{12} W_{21} T_{3}^{11}+T_{1}^{12} W_{22} T_{3}^{21} .
$$

Combining (3.11), (3.12), with (3.13), we get the following equality:

$$
\left(T_{1}^{11}\left(T_{2}^{11}\right)^{-1} T_{3}^{11}\right)\left(T_{1}^{11}\left(T_{2}^{11}\right)^{-1} T_{3}^{11}\right)^{\dagger}=\mu \mu^{\dagger}
$$

is valid for arbitrary bounded linear operators $W_{21}$ and $W_{22}$.

On the other hand, since $T_{1} T_{2}^{\dagger} T_{3} \neq O$, it follows that

$$
T_{1} T_{2}^{\dagger} T_{3}=\left(\begin{array}{cc}
T_{1}^{11}\left(T_{2}^{11}\right)^{-1} T_{3}^{11} & O \\
O & O
\end{array}\right) \neq O
$$

that is $T_{1}^{11}\left(T_{2}^{11}\right)^{-1} T_{3}^{11} \neq O$, which also implies that $T_{1}^{11} \neq O, T_{2}^{11} \neq O$ and $T_{3}^{11} \neq O$. Let $W_{22}=O$, then from (3.14), we obtain that the following equality:

$$
\begin{aligned}
& \left(T_{1}^{11}\left(T_{2}^{11}\right)^{-1} T_{3}^{11}\right)\left(T_{1}^{11}\left(T_{2}^{11}\right)^{-1} T_{3}^{11}\right)^{\dagger} \\
= & \left(T_{1}^{11}\left(T_{2}^{11}\right)^{-1} T_{3}^{11}+T_{1}^{12} W_{21} T_{3}^{11}\right)\left(T_{1}^{11}\left(T_{2}^{11}\right)^{-1} T_{3}^{11}+T_{1}^{12} W_{21} T_{3}^{11}\right)^{\dagger}
\end{aligned}
$$

holds for any $W_{21}$. This implies $T_{1}^{12}=O$. According to the fact: $R\left(T_{1}^{*}\right) \subseteq R\left(T_{2}^{*}\right) \Leftrightarrow$ $T_{1}^{12}=O$, it follows that $(1) \Rightarrow(2)$. $\square$

By Lemma 1.2 and Lemma 1.3, we know that for a bounded linear operator $T_{2}, X \in T_{2}\{1,4\}$ if and only $X^{*} \in T_{2}^{*}\{1,3\}$. So results for the operator product $T_{1} T_{2}^{(1,4)} T_{3}$ involving $\{1,4\}$-inverses of $T_{2}$ follow from the previous theorems in this section. 
TheOrem 3.4. Let $T_{1} \in L(\mathbb{L}, \mathbb{H}), T_{2} \in L(\mathbb{L}, \mathbb{K})$, and $T_{3} \in L(\mathbb{H}, \mathbb{K})$ be such that $T_{1}, T_{2}, T_{3}$ have closed ranges. Suppose that $T_{1}, T_{2}$, and $T_{3}$ are not zero operators on Hilbert spaces. Then the following statements are equivalent:

(1) The equality $T_{1} T_{2}^{(1,4)} T_{3}=T_{1} T_{2}^{\dagger} T_{3}$ holds for every $T_{2}^{(1,4)} \in T_{2}\{1,4\}$;

(2) $R\left(T_{3}\right) \subseteq R\left(T_{2}\right)$.

Corollary 3.5. Let $T_{1} \in L(\mathbb{L}, \mathbb{H}), T_{2} \in L(\mathbb{L}, \mathbb{K})$, and $T_{3} \in L(\mathbb{H}, \mathbb{K})$ be such that $T_{1}, T_{2}, T_{3}$ have closed ranges. Suppose that $T_{1}, T_{2}$, and $T_{3}$ are not zero operators on Hilbert spaces. Then the identity $T_{1} T_{2}^{(1,4)} T_{3}=O$ holds for every $T_{2}^{(1,4)} \in T_{2}\{1,4\}$ if and only if $R\left(T_{3}\right) \subseteq R\left(T_{2}\right)$ and $R\left(T_{3}\right) \subseteq N\left(T_{1} T_{2}^{\dagger}\right)$.

TheOrem 3.6. Let $T_{1} \in L(\mathbb{L}, \mathbb{H}), T_{2} \in L(\mathbb{L}, \mathbb{K})$, and $T_{3} \in L(\mathbb{H}, \mathbb{K})$ be such that $T_{1}, T_{2}, T_{3}$ have closed ranges. Suppose that $T_{1}, T_{2}$, and $T_{3}$ are not zero operators on Hilbert spaces and $T_{1} T_{2}^{\dagger} T_{3} \neq O$. Then the following statements are equivalent.

(1) $R\left(T_{1} T_{2}^{(1,4)} T_{3}\right)$ is the same for every $T_{2}^{(1,4)} \in B\{1,4\}$;

(2) $R\left(T_{3}\right) \subseteq R\left(T_{2}\right)$.

Acknowledgments. The authors would like to thank Prof. Oskar Maria Baksalary and the anonymous referees for their helpful suggestions, which greatly improved the quality of this paper.

\section{REFERENCES}

[1] J.K. Baksalary. A new approach to the concept of a strong unified-least squares matrix. Linear Algebra Appl., 388:7-15, 2004.

[2] J.K. Baksalary and O.M. Baksalary. An invariance property related to the reverse order law. Linear Algebra Appl., 410:64-69, 2005.

[3] J.K. Baksalary and R. Kala. Range invariance of certain matrix products. Linear Multilinear Algebra, 14:89-96, 1983.

[4] J.K. Baksalary and T. Pukkila. A note on invariance of the eigenvalues singular values, and norms of matrix products involving generalized inverses. Linear Algebra Appl., 165:125-130, 1992.

[5] A. Ben-Israel and T.N.E. Greville. Generalized Inverses: Theory and Applications. WileyInterscience, New York, 1974. Second Edition, Springer-Verlag, New York, 2003.

[6] R.H. Bouldin. The pseudo-inverse of a product. SIAM J. Appl. Math., 24:489-495, 1973.

[7] J.B. Conway. A Course in Functional Analysis. Springer-Verlag, New York, 1990.

[8] D.S. Cvetković-Ilić and R. Harte. Reverse order laws in $C^{*}$-algebras. Linear Algebra Appl., 434:1388-1394, 2011.

[9] D.S. Djordjević. Further results on the reverse order law for generalized inverses. SIAM J. Matrix. Anal. Appl., 29:1242-1246, 2007.

[10] A.M. Galperin and Z. Waksman. On pseudoinverse of operator products. Linear Algebra Appl., 33:123-131, 1980.

[11] C.W. Groetsch. Generalized Inverse of Linear Operators: Representation and Approximation. Marcel Dekker, Inc., New York, 1977. 
[12] J. Groß. Comment on range invariance of matrix products. Linear Multilinear Algebra, 41:157160,1996

[13] J. Gro $\beta$ and Y. Tian. Invariance properties of a triple matrix product involving generalized inverses. Linear Algebra Appl., 417:94-107, 2006.

[14] R.E. Harte. Invertibility and Singularity for Bounded Linear Operators. Marcel Dekker, Inc., New York, 1988

[15] M.Z. Nashed, editor. Generalized Inverse and Applications. Academic Press, New York-London, 1976.

[16] M.Z. Nashed. Inner, outer and generalized inverses in Banach and Hilbert spaces. Numer. Funct. Anal. Optim., 9:261-325, 1987.

[17] R. Penrose. A generalized inverses for matrices. Proc. Cambridge Philos. Soc., 51:406-413, 1955.

[18] Y.Y. Tseng. The Charateristic Value Problem of Hermitan Functional Operations in a NonHilbertian Space. Doctoral Dissertation in Mathematics, University of Chicago, 1933.

[19] Y.Y. Tseng. Generalized inverse of unbounded operators between two unitary spaces. Dokl. Akad. Nauk SSSR (N.S.), 67:431-434, 1949.

[20] Y.Y. Tseng. Properties and classification of generalized inverse of closed operators. Dokl. Akad. Nauk SSSR (N.S.), 67:607-610, 1949.

[21] Y.Y. Tseng. Sur les solutions des équations opératrices fonctionnelles entre les espaces. Unitaires, C. R. Acad. Sci. Paris, 228:640-641, 1949.

[22] Y.Y. Tseng. Virtual solutions and general inversions. Uspehi. Mat. Nauk (N.S.), 11:213-213, 1956.

[23] G. Wang, Y. Wei, and S. Qiao. Generalized Inverses: Theory and Computations. Science Press, Beijing-New York, 2004.

[24] Z.P. Xiong and Y.Y. Qin. Mixed-type reverse-order laws for the generalized inverses of operator products. Arab. J. Sci. Eng., to appear, DOI: 10.1007/s13369-011-0046-8. 